\title{
Preparation of Microspheres and Microfiber Membranes from Liquefaction Products of Salix Holocellulose by Electrospinning
}

Liping Jin, Yanfei Pan, Surigala, Dingwen Yin, Shuaiqi Hu, Haibiao Yu, Zhaoqi Wang, and Jintian Huang *

Salix holocellulose liquefaction products (SHLP) were prepared by liquefaction of the wood. A spinning solution of polyurethane (PU) and SHLP was prepared, and microspheres were obtained by electrospinning. The bonding of SHLP and PU-SHLP was characterized via FTIR. The conductivity and viscosity of spinning solution were characterized by conductivity meter and viscosity meter, respectively. The electrospun fiber morphologies were characterized by SEM. The results showed that PU was introduced into SHLP. The concentration of solution was the main factor that affected the morphology of microspheres, and the diameters of microspheres was from 0.18 to $1.18 \mu \mathrm{m}$. In addition, in this electrospinning system, the concentration of liquefied products in the mixed spinning solution was the major factor that affected the morphology of microfibers, and the diameters of microfibers were from 0.29 to $1.78 \mu \mathrm{m}$.

Keywords: Salix; Holocellulose; Microspheres; Microfiber membrane; Electrospinning

Contact information: College of Material Science and Art Design, Inner Mongolia Agriculture University, Hohhot 010018 China; *Corresponding author: jintian_h@163.com

\section{INTRODUCTION}

As a major agricultural country, China has abundant biomass resources and great potential for new energy development. Cellulose, as the most abundant renewable bioresource, has attracted significant attention for advanced materials and manufacturing in this epochal transition. Cellulose is one of the most abundant biomass resources in nature, and its hydrolysis and transformation to produce valuable chemicals are the focus of current research (Chen et al. 2020). A comprehensive explanation of sustainable functional materials and classical spinning techniques as key applications has been provided (Petroudy et al. 2021).

Electrospinning is a simple and effective technology used to prepare nanofiber materials (Hivechi et al. 2020). Nanofibrous membranes mainly fabricated by electrospinning techniques have gained substantial attention in the field of filtration-based membrane separation technology (Otsuka Issei and Barrett 2019). This is due to their unique morphological properties including small fiber diameter, high surface-to-volume ratio, high porosity, good mechanical properties, light weight, and accessible functionalization.

A microsphere is here defined as a micron bead on a nanofiber created via an electrospinning process of a polymer. Microspheres prepared by this method have shown high adsorption capacity, good selectivity, and excellent reusability for Bovine HemogloBin (BHb) (Liang et al. 2020). In addition, the manufacture of cellulose microspheres containing sulfonic acid groups by Chen (2020) was expected to adsorb and 
separate $\mathrm{Li}^{+}$ions in aqueous solution, so water resources polluted by heavy metal ions will be greatly improved. The incorporation of microspheres into the filter material increases the surface roughness of the nanofiber membranes. The size of the nanofibers inside the channel are larger, and thus the likelihood of harmful particles being retained when passed through the fiber membranes is reduced. The filtration efficiency is increased, and the filtration pressure of the material drops (Wang et al. 2008; Tijing et al. 2013). The surface roughness of the hydrophobic polyurethane nanofiber membrane increased because of the beads on the nanofibers (Li et al. 2017). Polyurethane nanofiber membranes have high biocompatibility (Thomas and Jayabalan 2009; Roohpour et al. 2010) and good mechanical properties (Heiny and Shastri 2016; Ju et al. 2017). In addition, the melting microspheres prepared by electrostatic spinning have large specific surface area and strong adsorption capacity, which is of great help to mitigate environmental pollution.

Holocellulose is a generic term for cellulose and hemicellulose, which is a part of wood. Holocellulose remains after the removal of lignins and resins in wood. Resins can be extracted using a mixture of benzene and alcohol as the solvent. The liquefaction products of holocellulose are more beneficial to the reaction of the polyurethane than the lignin-containing wood. This is because when the cellulose and lignin are liquefied in coexistence, the $\mathrm{C} 1$ position in the pyranose structure is replaced by a relatively strong phenolic light base in the lignin, which in turn produces a polycondensate residue that is hardly soluble in N,N-dimethylformamide (DMF) (Kobayashi et al. 2004). The condensation residue may affect the synthesis of the polyurethane.

Due to the high amount of hydrogen bonds in the cellulose molecules, the cellulose is more difficult to dissolve in common solvents. In electrospinning, the boiling point of the cellulose solvent affects the fiber collection effect (Kurokawa and Hotta 2019). If the boiling point is too high, the solvent is not easily volatilized and causes the fiber to adhere to the collecting plate (Kim et al. 2006; Yun et al. 2008). However, if the boiling point is too low, this leads to the solvent being very volatile and as a result the polymer blocks the spinneret. As a consequence, the electrospun cellulose has been difficult to prepare.

In this study, holocellulose was transformed into liquefaction products via liquefaction, and the process was used in the production of synthetic polyurethane with isocyanate. Polyurethane nano-materials can be prepared via electrospinning.

\section{EXPERIMENTAL}

\section{Materials}

Salix powder was collected from the Inner Mongolia Autonomous Region, Erdos City (China). Benzene, 95\% ethanol, glacial acetic acid, sulfuric acid, polyethylene glycol 400 (PEG-400), glycerol, and methanol were supplied by Tianjin Fengchuan Chemical Reagent Science and Technology Co., Ltd. (Tianjin, China). Sodium chlorite was purchased from Xiya Chemical Industry Co., Ltd. (Linyi, China). The 4,4diphenylmethane isocyanate (MDI) was supplied by Aladdin Reagent (Shanghai) Co., Ltd. (Shanghai, China). The N, N-dimethylformamide (DMF) and tetrahydrofuran (THF) were supplied by Tianjin Branch UNITA Chemical Industry and Trade Co., Ltd. (Tianjin, China). Lastly, the thermoplastic polyurethanes (TPU) 90A were supplied by Bayer Company in Leverkusen, Germany. 


\section{Preparation of Salix holocellulose}

First, $3 \mathrm{~g} \pm 0.2 \mathrm{~g}$ of Salix powder was wrapped with qualitative filter paper that was strapped by cotton thread. The extract solution was dispensed in a volume ratio of benzene to ethanol of 2:1, and the wrapped Salix powder was placed into the Soxhlet extractor in a $89{ }^{\circ} \mathrm{C}$ water bath for $6 \mathrm{~h}$. After the extraction, the filter paper bag of the sample was removed and air-dried.

Then, $2 \mathrm{~g}$ of the extracted Salix of wood and $65 \mathrm{~mL}$ of deionized water were added to a $250-\mathrm{mL}$ Erlenmeyer flask. Next, $0.5 \mathrm{~mL}$ of glacial acetic acid and $0.75 \mathrm{~g}$ of sodium chlorite were added into the Erlenmeyer flask each hour into a $75{ }^{\circ} \mathrm{C}$ water bath, and this was repeated four times. The sample was washed with deionized water until the filtrate was neutral and was washed with acetone three times, then oven-dried at $100{ }^{\circ} \mathrm{C}$ for $4 \mathrm{~h}$.

\section{Preparation of Salix holocellulose liquefaction products (SHLP)}

Five g of Salix holocellulose, $10 \mathrm{~g}$ of glycerol, $40 \mathrm{~g}$ of polyethylene glycol, and 0.5 $\mathrm{g}$ of sulfuric acid were added into a three-necked flask that was connected to a mechanical agitation and condensing device. The reaction system was heated in an oil bath at $170{ }^{\circ} \mathrm{C}$ and after $2 \mathrm{~h}$ the three-necked flask was placed in a room temperature water bath to cool down until the end of the experiment. The liquefied product was repeatedly washed with an acetone solution, and the filtrate was filtered until the filtrate became colorless to produce an insoluble residue and filtrate. The mixture of the acetone and liquefied product was then concentrated.

\section{Preparation of polyurethane of Salix holocellulose liquefaction products (PU-SHLP)}

An amount of $5 \mathrm{~g}$ of the concentrated liquefied product was vacuum pumped for 2 $\mathrm{h}$ in a $110{ }^{\circ} \mathrm{C}$ oil bath at $0.04 \mathrm{kPa}$. Then, it was added into a three-necked flask with a mechanical mixing system and nitrogen protection. Next, $1.5 \mathrm{~g}$ of 4, 4-diphenylmethane isocyanate (MDI), melted at $60{ }^{\circ} \mathrm{C}$ in the oven, was added to the flask, while the mechanical mixing equipment was working. The reaction system was heated in an $85^{\circ} \mathrm{C}$ oil bath for 1 h. After $1 \mathrm{~h}, 0.5 \mathrm{~g}$ of melted MDI was added into the system and heated for $30 \mathrm{~min}$. After $30 \mathrm{~min}$, another $0.5 \mathrm{~g}$ of MDI was added again. After $30 \mathrm{~min}$, the oil bath temperature was reduced to $65^{\circ} \mathrm{C}$ while excess anhydrous methanol was added into the reaction system and allowed the reaction to run for $1 \mathrm{~h}$. The product was transferred out and filled with nitrogen.

\section{Electrospinning of $P U-S H L P$}

First, $2 \mathrm{~g}$ of polyurethane Salix holocellulose liquefaction products were added into $38 \mathrm{~g} \mathrm{DMF}$ (w1: $\mathrm{w} 2=5 \%$ ), and the system was stirred. The solutions of 10\%, 15\%, and $20 \%$ were configured in the same way.

The spinning solutions were charged into a syringe and mounted on a self-made electrospinning machine with a spinning voltage of $27.15 \mathrm{kV}$ and the distance from the syringe needle to receiving plate was set at a distance of $12 \mathrm{~cm}$.

\section{Electrospinning of TPU and SHLP}

Spinning solutions were prepared by dissolving TPU $(20 \mathrm{wt} \%)$ and the salix holocellulose liquefaction product $(25,50$, and $75 \mathrm{wt} \%$ of TPU mass) in DMF and THF (w:w=1:1) with vigorous stirring over $4 \mathrm{~h}$.

The spinning solutions were charged into a syringe and mounted on a self-made electrospinning machine with a spinning voltage of $15 \mathrm{kV}$. The distance from the syringe needle to receiving plate was set at a distance of $12 \mathrm{~cm}$. 


\section{Methods}

Fourier transform infrared (FTIR) characterization

The SHLP and the polyurethane from the SHLP were characterized using a Nicolet Magna-IR 750 FTIR spectrometer (Madison, WI, USA).

\section{Measurement of conductivity of spinning solution}

The conductivity of the spinning solution was measured using a DDS-11A conductivity meter produced by INESA Scientific Instrument Co., Ltd. (Shanghai, China).

\section{Measurement of viscosity of spinning solution}

The viscosity of the spinning solution was measured using a NDJ-5S viscosity meter produced by Shanghai Yueping Scientific Instrument Co., Ltd. (Shanghai, China).

Scanning electron microscopy (SEM)

The electrospun fiber morphologies of the polyurethane synthesized from SHLP and the liquefaction products with TPU were characterized using a scanning electron microscope (S-3400N, Hitachi, Tokyo, Japan). Using these SEM images, 100 fibers were randomly selected, and the average diameter was calculated.

\section{RESULTS AND DISCUSSION}

\section{FTIR of Salix Holocellulose and Liquefaction Products}

The FTIR spectra of the Salix holocellulose and the liquefaction products are shown in Fig. 1.

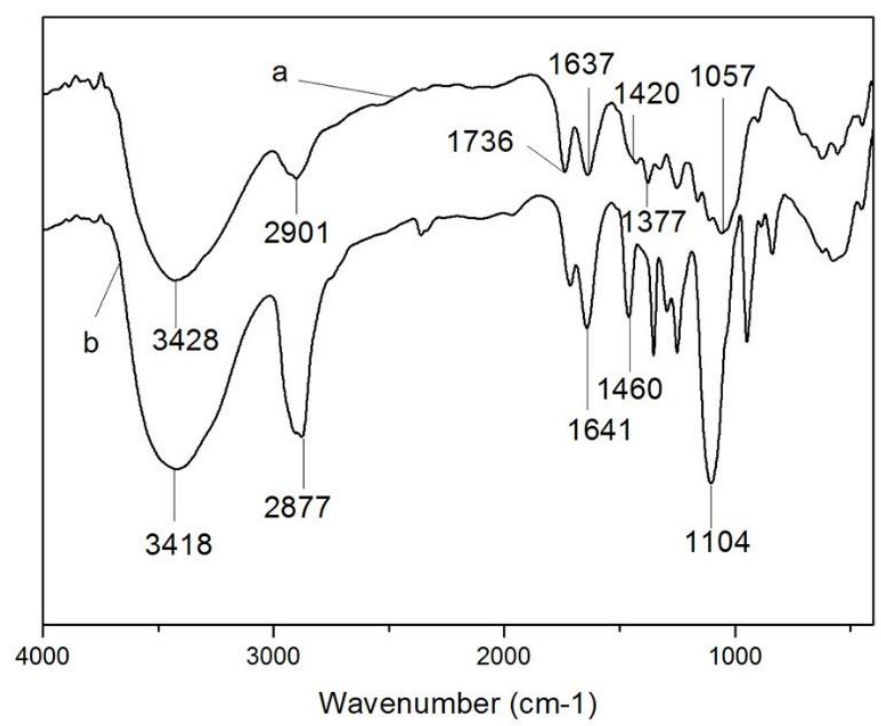

Fig. 1. FTIR spectra of the Salix holocellulose (a) and the liquefaction products (b)

The pair of FTIR spectra, before and after liquefaction of the salix holocellulose, revealed obvious changes (Bilba and Ouensanga 1996; Pandey 1999). The absorption peak at $3428 \mathrm{~cm}^{-1}$ indicated the telescopic vibration of $\mathrm{O}-\mathrm{H}$, and after the liquefaction reaction, more $\mathrm{O}-\mathrm{H}$ was exposed. Therefore, the peak moved to $3418 \mathrm{~cm}^{-1}$, and the peak shape 
became broad. In the FTIR spectra of the Salix holocellulose, the absorption peak was at $2901 \mathrm{~cm}^{-1}$ and represented the telescopic vibration of $-\mathrm{CH}$ and $-\mathrm{CH}_{3}$. After the liquefaction reaction, this peak moved to $2877 \mathrm{~cm}^{-1}$, the absorption intensity became larger, and the peak shape became sharp. This may explain how the holocellulose chains in the acid were catalyzed $\left(\mathrm{H}^{+}\right)$under the action of the fracture and how the macromolecules of the holocellulose were degraded into small molecules. From $1736 \mathrm{~cm}^{-1}$ to $500 \mathrm{~cm}^{-1}$, the shapes of the respective absorption peaks of the liquefaction product were sharper than the characteristic peaks of the non-reactive holocellulose, indicating that the functional groups representing the peaks were more exposed. It was possible that the active groups on small molecules were also increasing. Absorbance peaks at $1057 \mathrm{~cm}^{-1}, 1420 \mathrm{~cm}^{-1}$, and $1637 \mathrm{~cm}^{-1}$ corresponded to absorbed Aryl C-H plane deformation, C- $\mathrm{H}$ plane deformation, and $\mathrm{H}_{2} \mathrm{O}$ stretching vibration (Pan et al. 2016; Hao et al. 2018). The whole cellulose liquefaction process needs a high temperature of $140{ }^{\circ} \mathrm{C}$. Under high temperature the hydrogen bonds on the whole cellulose molecular chain will break to some extent, resulting in the destruction of hydrogen bonds and the fracture of cellulose molecular chain. Therefore, large molecules of holocellulose were degraded to small molecules. The changes in the typical absorption peaks were as follows. The peak of $1637 \mathrm{~cm}^{-1}$ moved to $1641 \mathrm{~cm}^{-1}$, indicating that the absorption peak of the holocellulose was an ion sample. The peak at $1420 \mathrm{~cm}^{-1}$ moved to $1460 \mathrm{~cm}^{-1}$, showing the bending vibration of $-\mathrm{CH}$ and $-\mathrm{CH}_{2}$. The peak at $1377 \mathrm{~cm}^{-1}$ moved to $1352 \mathrm{~cm}^{-1}$, indicating this was the characteristic peak of cellulose and the bending vibration of $-\mathrm{CH}$ in aliphatic; and the peak at $1057 \mathrm{~cm}^{-1}$ moved to 1104 $\mathrm{cm}^{-1}$, which was the C-O stretching vibration of alcohols, indicating the large release of alcohol after liquefaction (Zhang and Huang 2006; Zhou et al. 2015).

\section{FTIR Characterization of PU-SHLP}

In Fig. 2, the broad peak near $3396 \mathrm{~cm}^{-1}$ was the stretching vibration peak of the $\mathrm{N}-\mathrm{H}$ group participating in the hydrogen-bonding carbamate, while the peak at $2948 \mathrm{~cm}^{-1}$ was the methylene stretching vibration.

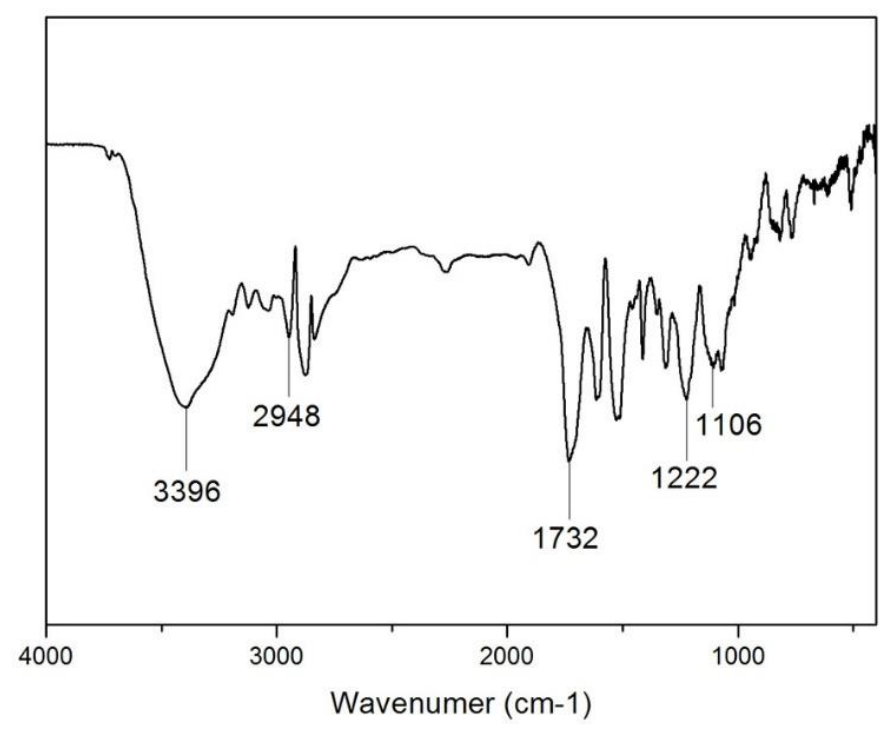

Fig. 2. FTIR spectra of PU-SHLP 
The absorption peak at $1732 \mathrm{~cm}^{-1}$ indicated the stretching vibration absorption peak that belonged to the "amide I" peak. The peak at $1222 \mathrm{~cm}^{-1}$ was attributed to the $=\mathrm{C}-\mathrm{O}-\mathrm{C}$ stretching vibration of the carbamate. These were the infrared characteristic peaks of the polyurethane, which indicated that the liquefaction products synthesized the polyurethane with the MDI. The peak at $1106 \mathrm{~cm}^{-1}$ was the vibration telescopic absorption peak of the ether bond -C-O-C, where the sample was a polyether type polyurethane (Ang et al. 2014; Laurichesse et al. 2014).

\section{Effect of Concentration of the Spinning Solution on the Morphology of Electrospun PU-SHLP}

To allow the PU synthesized from the liquefied products to be electrospun, four different concentrations of the liquefied polyurethane solution were configured. The percentages $5 \%, 10 \%, 15 \%$, and $20 \%$ referred to liquefied polyurethane (LP). The LP was dissolved in organic solvents, thereby made into spinning liquids. Therefore, four samples were selected as the analyzed objects of electrospinning. Their concentrations were 5\%, $10 \%, 15 \%$, and $20 \%$, respectively. Spinning the solution formed electrostatic spraying after applying high voltage. It showed that a circle of small droplets were along the bottom of needle. The difference in the four solutions was that the ejected droplets of $5 \%$ and $10 \%$ were larger than those of $15 \%$ and $20 \%$, and the jets of $5 \%$ and $10 \%$ were unstable and irregularly scattered on the receiving plate.

Table 1. Characteristics of the Different Concentrations of the Spinning Solution and Electrospun Fibers

\begin{tabular}{|c|c|c|c|c|c|}
\hline Solvent & $\begin{array}{c}\text { PU- } \\
\text { SHLP\% }\end{array}$ & Conductivity $(\mathrm{mS})$ & Viscosity $(\mathrm{cp})$ & Morphology & Diameter $(\mu \mathrm{m})$ \\
\hline DMF & 5 & 5 & 0.153 & globular & 0.64 \\
\hline DMF & 10 & 5 & 0.271 & globular & 0.45 \\
\hline DMF & 15 & 8 & 0.425 & globular & 0.59 \\
\hline DMF & 20 & 10 & 0.622 & globular & 0.40 \\
\hline
\end{tabular}

The morphology of the electrospun Salix holocellulose liquefaction product structures is shown in Fig. 3.
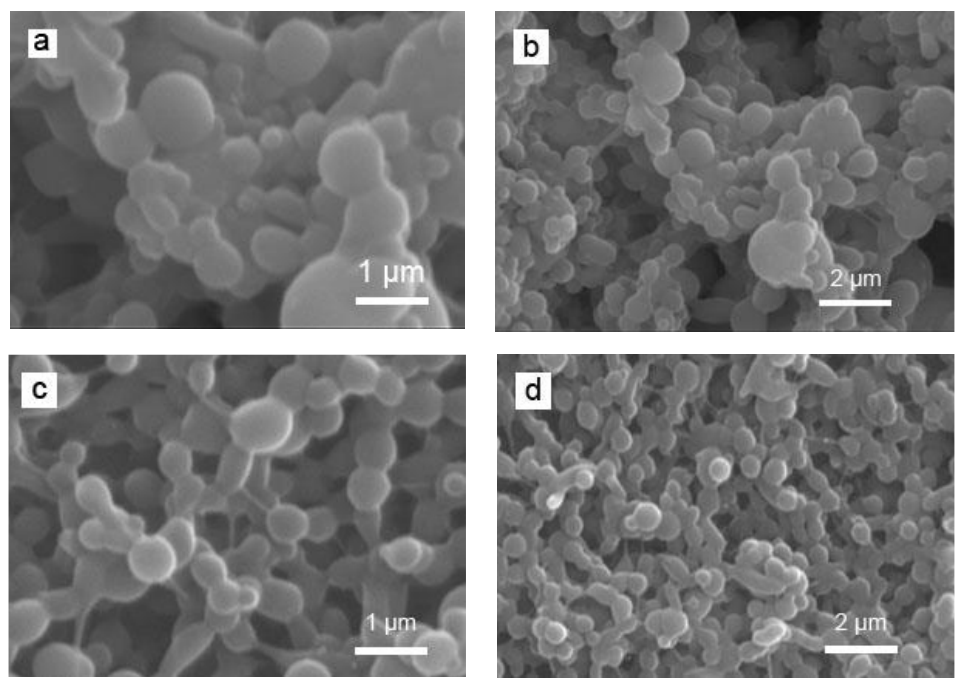

Fig. 3. SEM images of the electrospun fibers obtained from PU-SHLP solutions at different PUSHLP concentrations: (a)(b) $15 \%$ and (c)(d) $20 \%$ 
Figure 3 shows that the $15 \%$ concentration solutions provided irregular beads that were stacked together via electrospinning. The diameter of each bead was in the range of $0.18 \mu \mathrm{m}$ to $1.18 \mu \mathrm{m}$ with a mean diameter of $0.59 \mu \mathrm{m}$. The $20 \%$ concentration solutions provided more independent beads than the $15 \%$ solution. The diameter of the minimum bead was in the range of $0.17 \mu \mathrm{m}$ to $0.66 \mu \mathrm{m}$ with a mean diameter of $0.40 \mu \mathrm{m}$. These beads were nonbonding. Under the same processing conditions of electrospinning, two different morphologies of the beads appeared, with the most likely reason being the different concentrations of the spinning solutions. The solvent quality of the $15 \%$ solution was greater than the $20 \%$ solution. During electrospinning, the solvent evaporation rate of the $15 \%$ solution was less than the $20 \%$ solution. This led to excess solvent that could not be evaporated in time, which mixed with the beads. The morphology of the $15 \%$ solution was such that the beads stacked together. In the SEM images of the $20 \%$ solution, a few filamentous fibers were observed between the beads. The solution viscosity depended mainly on the concentration of the solution and the molecular weight of the polymer. A higher solution concentration resulted in greater viscosity, and when the polymer mass fraction increased, the solution viscosity also increased. As the solution viscosity increased, the charge density on the jet surface increased, at which point the jet whirl instability dominated and reduced the fiber diameter. It has been found that as the diameter increases, the distance between the beads becomes longer, and the beads change from a spherical shape to fusiform (Zhang et al. 2005; Ye et al. 2006; Costa et al. 2010).

\section{Effect of Different Concentrations of the SHLP in Solutions on the Morphology of Electrospun TPU and SHLP}

From the photos, all three mass fractions of the spinning solution could form films via electrospinning. These films had enough mechanical strength to allow them to easily peel off from the surface of the receiving board completely. The colors of the films became darker as the mass fraction of SHLP was increasing, as shown in Fig. 4.

Table 2. Characteristics of the Different Concentrations of the Spinning Solution and Electrospun Fibers

\begin{tabular}{|l|c|c|c|c|c|}
\hline Solvent & $\begin{array}{c}\text { PU- } \\
\text { SHLP\% }\end{array}$ & $\begin{array}{c}\text { Conductivity } \\
(\mathrm{S} / \mathrm{cm})\end{array}$ & $\begin{array}{c}\text { Viscosity } \\
(\mathrm{CP})\end{array}$ & Morphology & $\begin{array}{c}\text { Diameter } \\
(\mu \mathrm{m})\end{array}$ \\
\hline \multirow{3}{*}{ DMF+THF } & 25 & 16 & 0.624 & Fiber & 0.44 \\
\cline { 2 - 6 } & 50 & 12 & 1.033 & Fiber & 1.23 \\
\cline { 2 - 6 } & 75 & 10 & 1.181 & Hole & 1.58 \\
\hline
\end{tabular}
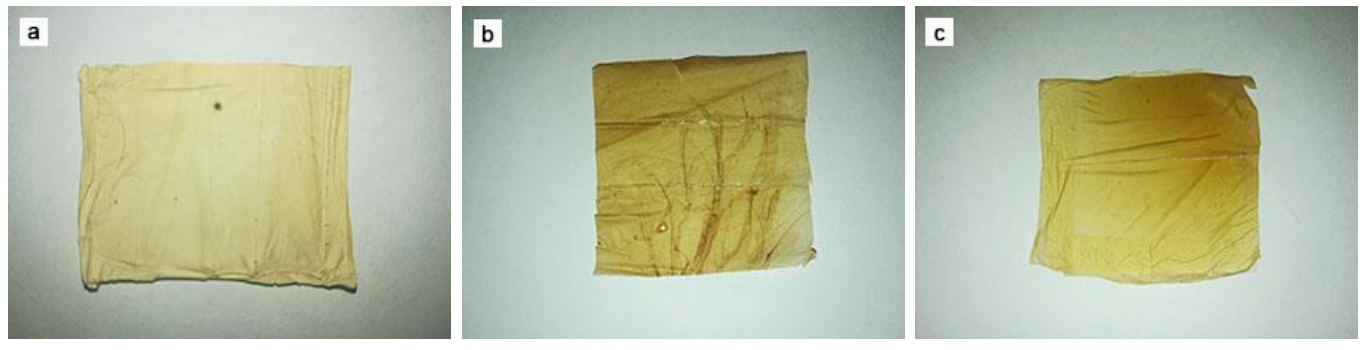

Fig. 4. Photographs of films of different concentrations of the SHLP in solutions of TPU90A and SHLP via electrospinning: (a) $25 \%$; (b) $50 \%$; and (c) $75 \%$ 

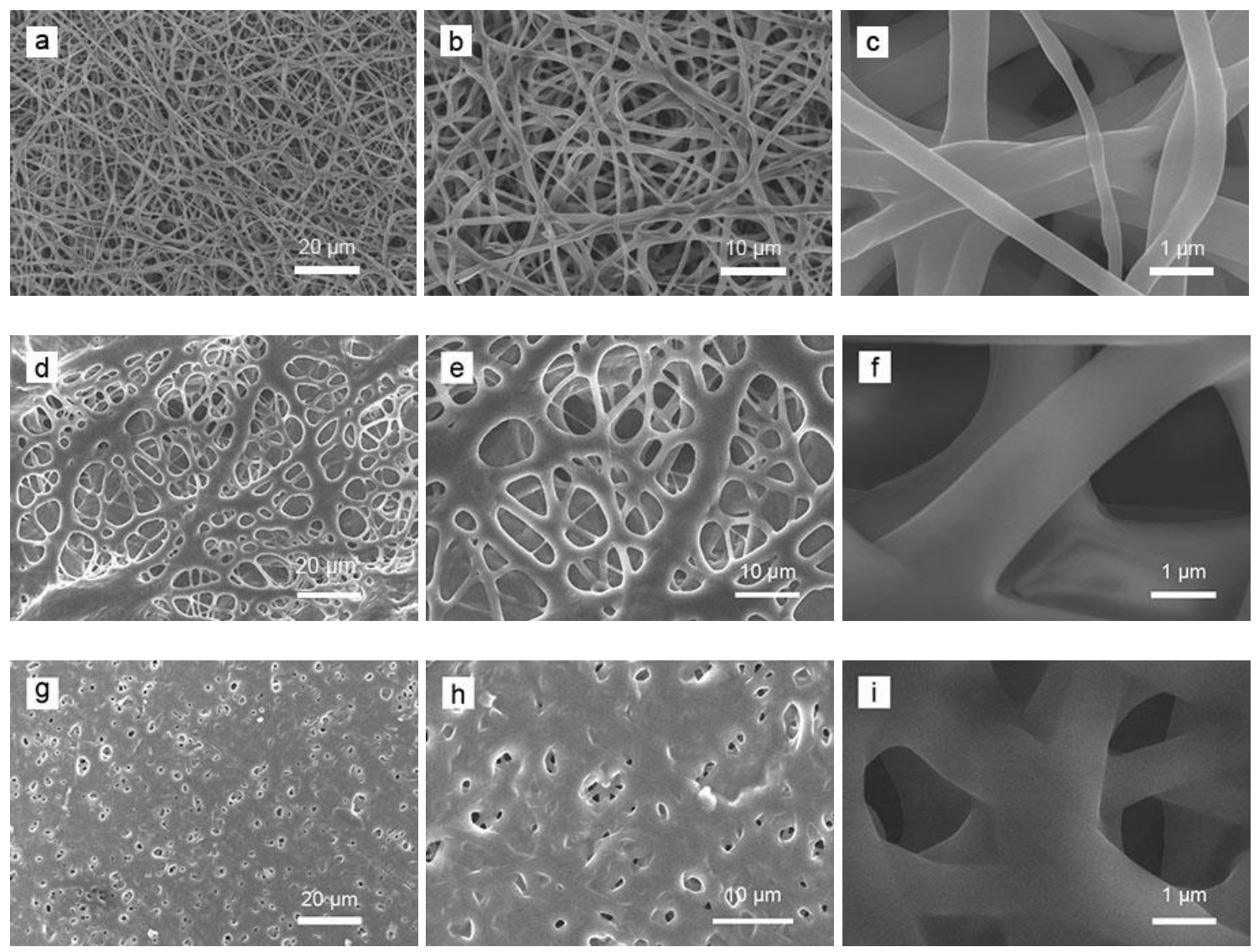

Fig. 5. SEM images of the electrospun fibers obtained from SHLP in the TPU90A solutions at different SHLP concentrations: (a)(b)(c) 25\%; (d)(e)(f) 50\%; and (g) (h) (i) $75 \%$

Figure 5 shows a considerable difference in the microstructures between the fibers obtained via electrospinning. In Fig. 5(a), the fibers showed a typical micro-nano fiber shape, long and straight, and the diameters of the fibers were more uniform. The fibers were intertwined, but the micro-nano morphology was clearly observed. In Fig. 5(d), the diameters of the fibers were greater in length and were shorter than those in Fig. 5(a). The fibers adhered to each other and reformed holes. In Fig. 5(g), the fiber's adhesion was more severe than the ones of the $50 \%$ solution; hence the diameters of holes decreased and the holes almost disappeared.

Salix holocellulose liquefaction products are polyols. They did not cross-link, and they could only be attached to the TPU for electrospinning to become micro-nano fibers. The addition of Salix holocellulose liquefaction products increased the solvent mass of the TPU solution. Therefore, the TPU relative mass fraction of the spinning solution changed.

As a result, the $25 \%$ SHLP and TPU spinning solution was the least affected, and thus the micro-nanofibrous morphology was present in Fig. 5(a). The diameter of the microfibers had a range of 0.29 to $1.78 \mu \mathrm{m}$, and the mean diameter was $0.86 \mu \mathrm{m}$. The diameter of the nanofibers at $50 \%$ had a range of 0.31 to $8.67 \mu \mathrm{m}$ with a mean diameter of $1.8 \mu \mathrm{m}$. With the increase of the mass fraction of the SHLP in the spinning solution, the amount of solvent was reduced. In the same amount of electrostatic spinning time, the solvent's premature evaporation resulted in a shorter time for the fibrosis formation process. Simultaneously, the TPU concentration was increased and the surface tension of the TPU solution increased. The resulting TPU could not overcome the increased surface tension. 
Thus, the TPU failed to draw into fibers, and retracted into a spherical or hemispherical shape. Due to the lack of cross-linking from the SHLP, it could only be attached to the surface of the TPU fibers. The SHLP increased the diameter of the fibers, as shown in Fig. 5(d) and the fibers were stuck onto a sheet, as shown in Fig. 5(g). There were no microfibers observed except for the holes in Fig. 5(g). However, in Fig. 5(i), independent micro-nano fibers were still observed. With the increase of the mass fraction of SHLP in the spinning solution, the microstructure of the electrospinning showed a filament fibrous shape, a halffilament/half-pore shape, and the dense hole appearing in the image was the lamellar membrane.

\section{CONCLUSIONS}

1. The results of FTIR analysis showed that alcohol hydroxyl value of Salix holocellulose liquefaction products was $297.65 \mathrm{mg} \mathrm{KOH} / \mathrm{g}$, which provided the condition of synthesis for polyol and polyurethane (PU). Besides, there were characteristic peaks of PU, which indicated that Salix liquefaction products were involved in the synthesis of PU.

2. Microfiber could not be prepared from the spinning solution (the concentration is from $5 \%$ to $20 \%$ ) of PU prepared from the Salix holocellulose liquefaction products. Smooth microspheres could be prepared by $20 \%$ spinning solution. The average diameter of microspheres was up to $0.40 \mu \mathrm{m}$.

3. Microfiber membranes of Salix holocellulose liquefaction products were prepared by electrospinning with thermoplastic polyurethanes (TPU). The morphology of fibers prepared with $25 \%$ Salix holocellulose liquefaction products in $20 \%$ TPU solution was good. The average diameter was $0.86 \mu \mathrm{m}$ and they had typical microfiber characteristics.

\section{ACKNOWLEDGMENTS}

This work was supported by the National Key Technology Research and Development Program of the Ministry of Science, the Technology of China under Grant No. 2015BAC06B02-3, Natural Science Foundation of Inner Mongolia Autonomous Region under Grant No. 2019BS03014 and 2016MS0353, and Science and Technology Innovation Leading project of Inner Mongolia Autonomous Region (KCBJ2018013).

\section{REFERENCES CITED}

Ang, K. P., Lee, C. S., Cheng, S. F., and Cheng, H. C. (2014). "Synthesis of palm oilbased polyester polyol for polyurethane adhesive production," Journal of Applied Polymer Science 131(6), 596-602. DOI: 10.1002/app.39967

Bilba, K., and Ouensanga, A. (1996). "Fourier transform infrared spectroscopic study of thermal degradation of sugar cane bagasse," Journal of Analytical \& Applied Pyrolysis 38(1-2), 61-73. DOI: 10.1016/0144-4565(90)90070-Z

Chen, X. X., Tian, L. Y., Jing P., Long Z., and Mao, L. Z. (2020). "Efficient adsorption performance of lithium ion onto cellulose microspheres with sulfonic acid groups," Quantum Beam Science 4(1), 6. DOI: 10.3390/qubs4010006 
Costa, L. M. M., Bretas, R. E. S., and Gregorio, R. (2010). "Effect of solution concentration on the electrospray/electrospinning transition and on the crystalline phase of PVDF," Journal of Materials Sciences \& Applications 1(1), 247-252. DOI: 10.1.1.970.1022

Chen, Z. Y. (2020). "Review of flexible strain sensors based on cellulose composites for multi-faceted applications," Cellulose. DOI: 10.1007/s10570-020-03543-6

Hao, Y. N., Pan, Y. F., and Du, R. (2018). The influence of a thermal treatment on the decay resistance of wood via FTIR analysis," Advances in Materials Science and Engineering. 2018. DOI: 10.1155/2018/8461407

Heiny, M., and Shastri, V. P. (2016). "Nanofibers of elastin and hydrophilic segmented polyurethane solution blends show enhanced mechanical properties through intermolecular protein-polymer H bonding," Biomacromolecules 17(4) 1312-1320. DOI: 10.1021/acs.biomac.5b01681

Hivechi, A., Bahrami, S. H., and Siegel, R. A. (2020)." In vitro and in vivo studies of biaxially electrospun poly(caprolactone)/gelatin nanofibers, reinforced with cellulose nanocrystals, for wound healing applications," Cellulose 27(9), 5179-5196. DOI: $10.1007 / \mathrm{s} 10570-020-03106-9$

Ju, J., Shi, Z., Deng, N., Liang, Y., Kang, W., and Cheng, B. (2017). “Designing waterproof breathable material with moisture unidirectional transport characteristics based on a TPU/TBAC tree-like and TPU nanofiber double-layer membrane fabricated by electrospinning," RSC Advances 7(51), 32155-32163. DOI: 10.1039/c7ra04843b

Kim, C. W., Kim, D. S., Kang, S. Y., Marquez, M., and Yong, L. J. (2006). "Structural studies of electrospun cellulose nanofibers," Polymer 47(14), 5097-5107. DOI: 10.1016/j.polymer.2006.05.033

Kobayashi, M., Asano, T., Kajiyama, M., and Tomita, B. (2004). "Analysis of residue formation during wood liquefaction with polyhydric alcohol," Journal of Wood Science 50(5), 407-414. DOI: 10.1007/s10086-003-0596-9

Kurokawa, N., and Hotta, A. (2019). "Regenerated cellulose nanofibers fabricated through electrospinning and saponification of cellulose acetate as reinforcement of polylactide composites," Cellulose. 26(13), 7797-7808.

DOI: $10.1007 / \mathrm{s} 10570-019-02623-6$

Laurichesse, S., Huillet, C., and Averous, L. (2014). “Original polyols based on organosolv lignin and fatty acids: New bio-based building blocks for segmented polyurethane synthesis," Green Chemistry 16(8), 3958-3970.

DOI: $10.1039 / \mathrm{c} 4 \mathrm{gc00596a}$

Liang, C., Zhao, L. S., and Li, S. S. (2020). "Direct preparation of porous cellulose microspheres via a self-growth process on bamboo fibers and their functionalization for specific adsorption of histidine-rich proteins," Journal of Chromatography A 1633. DOI:10.1016/j.chroma.2020.461636

Li, T., Xu, Y. H., and Zou, F. Y. (2017). "Preparation and characterization of hydrophobic polyurethane nanofiber membrane by dual electrospinning," (http://kns.cnki.net/kcms/detail/33.1249.TS.20171019.1150.014.html), Accessed 19 Oct 2017.

Otsuka, I., and Barrett, C. J. (2019). "Electrospinning of photo-responsive azo-cellulose: towards smart fibrous materials," Cellulose 26(11). 6903-6915.

DOI: $10.1007 / \mathrm{s} 10570-019-02585-9$ 
Pan, Y. F., Guo, Z. Q., and Guo, T. C. (2016). "The preparation, characterization, and influence of multiple electroless nickel-phosphorus (Ni-P) hollow composite coatings on micro-nanocellulose fibers," Surface \& Coatings Technology 298, 33-38.

DOI:10.1016/j.surfcoat.2016.01.06

Petroudy, S. R. D, Kahagh, S. A., and Vatankhah, E. (2021). "Environmentally friendly superabsorbent fibers based on electrospun cellulose nanofibers extracted from wheat straw" Carbohydrate Polymers 251. DOI: 10.1016/j.carbpol.2020.117087

Roohpour, N., Wasikiewicz, J. M., Moshaverinia, A., Paul, D., Grahn, M., Rehman, I., and Vadama P. (2010). "Polyurethane membranes modified with isopropyl myristate as a potential candidate for encapsulating electronic implants: A study of biocompatibility and water permeability," Polymers 2(3), 102-119. DOI: $10.3390 /$ polym2030102

Thomas, V., and Jayabalan, M. (2009). "A new generation of high flex life polyurethane urea for polymer heart valve--studies on in vivo biocompatibility and biodurability," J. Biomed. Mater. Res. Part A 89A (1), 192-205. DOI: 10.1002/jbm.a.31937

Tijing, L. D., Choi, W., Jiang, Z., and Amarjargal, A. (2013). "Two-nozzle electrospinning of (MWNT/PU)/PU nanofibrous composite mat with improved mechanical and thermal properties," Current Applied Physics 13(7) 1247-1255. DOI: 10.1016/j.cap.2013.03.023

Ye, P., Xu, Z. K., Wu, J., Innocent, C., and Seta, P. (2006). "Nanofibrous membranes containing reactive groups: Electrospinning from poly (acrylonitrile-co-maleic acid) for lipase immobilization," Macromol. 39(3), 1041-1045. DOI: 10.1021/ma0517998

Yun, G. Y., Kim, H. S., Kim, J., Kim, K., and Yang, C. (2008). "Effect of aligned cellulose film to the performance of electro-active paper actuator," Sensors and Actuators A: Physical 141(2), 530-535. DOI: 10.1016/j.sna.2007.10.014

Zhang, Y., Ouyang, H., Lim, C. T., Ramakrishna, S., and Huang, Z. M. (2005). "Electrospinning of gelatin fibers and gelatin/PCL composite fibrous scaffolds," Journal of Biomedical Materials Research Part B: Applied Biomaterials 72B (1), 156-165. DOI: 10.1002/jbm.b.30128

Zhang, C. X., and Huang, J. T. (2006). "Liquefaction and FTIR analysis of two desert shrubs Salix psammophila and Caragana intermedia," Forestry Studies in China 8(3) 38-42. DOI: 10.1007/s11632-006-0022-7

Article submitted: November 6, 2017; Peer review completed: January 20, 2018; Revised version received and accepted: December 15, 2020; Published: December 22, 2020.

DOI: $10.15376 /$ biores.16.1.1245-1255 